\title{
MULTIVARIATE BAYES REGRESSION MODELS FOR SMOOTHING OF COLOR IMAGES
}

\author{
Saeko Kusanobu *
}

\begin{abstract}
We propose a smoothing method for color images based on multivariate Bayes models. This method simultaneously smoothes the images of red, green and blue lights while taking into account their correlations. The performance of the color smoothing technique is applied to test images and the Landsat TM data. We compare the multivariate smoothing with some other techniques which remove noise in monochrome images.
\end{abstract}

\section{Introduction}

We consider the multivariate model for noisy data $z_{i}=\left(z_{1 i}, \ldots, z_{p i}\right)^{\prime}, i=1, \ldots, n$ such that $z_{i}=\boldsymbol{\mu}_{i}+\boldsymbol{\epsilon}_{i}$, where we assume that the unknown mean vector $\boldsymbol{\mu}_{i}=\left(\mu_{1 i}, \ldots, \mu_{p i}\right)^{\prime}$ smoothly changes and that the error $\epsilon_{i}$ is a sample from a multivariate normal population with mean zero and variance $\Sigma$. The smoothing method such as nonparametric regression performs favorably when the true trend changes gradually. The multivariate methods through splines are given by Karlin, Micchelli and Rinott (1986) and Stone (1994). When the exploratory variable $i$ is equi-spaced, the model using the difference is useful. Hence, we propose the multivariate Bayes model using a difference matrix as a smoothness prior in Section 2.

The multivariate model for $p=3$ can be applied to smoothing of color images. We show the performance of multivariate smoothing for a noisy test image and the actual image obtained by the satellite, Landsat 5 in Section 3 . The images by the Landsat thematic mapper (TM) sensor have stripe noise along the horizontal direction, due to the sensoring system. TM images are geometrically rectified, but stripe noise is still observed in the area where the ratio of signal to noise is small such as water area. Pan and Chan (1992) employed a filtering technique with fast Fourier transform (FFT) for removing stripe noise. Nishii, Yanagimoto and Kusanobu (1997) applied a univariate Bayes linear model. These are methods for a monochrome image. On the other hand, our procedure simultaneously smoothes three monochrome images while taking into account their correlations. It is compared with the methods for the monochrome image, such as the univariate Bayes model, lowpass filtering and median filtering. The last section describes the features and the usefulness of our procedure.

\footnotetext{
${ }^{*}$ Graduate School of Engineering, Hiroshima University, Kagamiyama 1-4-1, Higashi-Hiroshima 739 8527, Japan,

Key words: Difference matrix, Landsat images, Smoothness prior
} 


\section{Bayes linear models}

Consider a serial data $\left(i, \boldsymbol{z}_{i}\right), i=1, \ldots, n$ where $\boldsymbol{z}_{i}=\left(z_{1 i}, \ldots, z_{p i}\right)^{\prime}$. We assume that $z_{i}$ is a sample from a multivariate normal population with mean $\boldsymbol{\mu}_{i}=\left(\mu_{1 i}, \ldots, \mu_{p i}\right)^{\prime}$ and variance-covariance matrix $\Sigma$. We define some notation before describing the model. Suppose that $\nabla$ is the first order difference as $\nabla u_{j}=u_{j+1}-u_{j}$, then the second and the third order differences are defined by the successive difference $\nabla^{r} u_{j}=\nabla\left(\nabla^{r-1} u_{j}\right)$ with $r=2,3$. Next, define $n \times n$ matrix $D_{r}(r=1,2,3)$ based on the $r$-th order difference as

$$
\boldsymbol{u}^{\prime} D_{r} \boldsymbol{u}=\sum_{j=1}^{n-r}\left(\nabla^{r} u_{j}\right)^{2}
$$

for the vector $\boldsymbol{u}=\left(u_{1}, \ldots, u_{n}\right)^{\prime}$. For example, $D_{1}$ and $D_{2}$ are expressed by

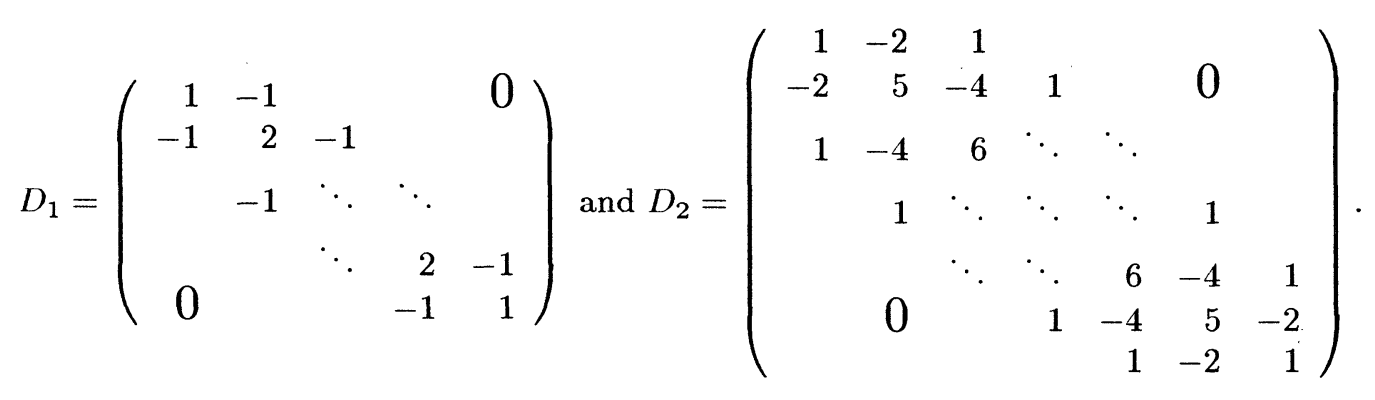

We note that the difference matrix $D_{r}$ is a positive semi-definite matrix of rank $n-r$ from the relation (1).

Now, let $Z=\left(z_{1}^{\prime}, \cdots, \boldsymbol{z}_{n}^{\prime}\right)^{\prime}$ and $M=\left(\boldsymbol{\mu}_{1}^{\prime}, \cdots, \boldsymbol{\mu}_{n}^{\prime}\right)^{\prime}$ be random matrices of size $n \times p$, and define the vectorization of matrix $Z$ such as $\operatorname{vec}(Z)=\left(z_{11}, \ldots, z_{1 n}, \ldots, z_{p 1}, \ldots, z_{p n}\right)^{\prime}$. We consider three prior distributions for $M$. Let $r$ be a fixed integer 1,2 or 3 . Then, assume the following Bayes linear model for $Z$ based on the $r$-th order,

$$
\operatorname{vec}(Z) \mid M \sim N_{p n}\left(\operatorname{vec}(M), \Sigma \otimes I_{n}\right) \text { and } \operatorname{vec}(M) \sim N_{p n}\left(\operatorname{vec}\left(F_{r} \Gamma\right), \tau^{2} \Sigma \otimes D_{r}^{-}\right),
$$

where $D_{r}^{-}$denotes a generalized inverse of $D_{r}, \Gamma$ and $F_{r}$ are a regression coefficient matrix and an $n \times r$ matrix $(r=1,2,3)$ composed by

$$
F_{r}^{\prime}=\left(f_{1}, \ldots, f_{r}\right), \text { where }\left\{\begin{array}{l}
f_{1}=\frac{1}{\sqrt{n}} e_{1}, f_{2}=\frac{\sqrt{12}}{\sqrt{n\left(n^{2}-1\right)}}\left(e_{2}-\frac{n+1}{2} e_{1}\right) \\
f_{3}=\frac{\sqrt{180}}{\sqrt{n\left(n^{2}-1\right)\left(n^{2}-4\right)}}\left\{e_{3}-(n+1) e_{2}+\frac{(n+1)(n+2)}{6} e_{1}\right\}
\end{array}\right.
$$

with $e_{k}=\left(1^{k-1}, 2^{k-1}, \ldots, n^{k-1}\right)^{\prime}$. The vectors $f_{k}, k=1,2,3$ are orthonormal eigenvectors of $D_{r}$ corresponding to the zero eigenvalue. Remaining positive eigenvalues are denoted by $\lambda_{r, r+1}<\cdots<\lambda_{r, n}$.

In these Bayes models, we assume that $\boldsymbol{\mu}_{i}$ changes smoothly in the exploratory variable $i$ and that smoothness is evaluated by the small squared sum of the $r$-th order differences. Moreover, for a fixed variate $j=1,2, \ldots, p$, the observations $z_{j 1}, \ldots, z_{j n}$ are distributed around a polynomial regression curve in the $(r-1)$-st order degree. We take into account the correlations $\Sigma$ of the $i$-th observations $z_{i}$. 
The entire likelihood $L_{r}(\Gamma, \delta, \Sigma)$ of $Z$ and $M$ of the models (2) is written by

$$
\delta^{\frac{(n-r) p}{2}}|2 \pi \Sigma|^{-\frac{2 n-r}{2}} \prod_{i=r+1}^{n} \lambda_{r, i}^{\frac{p}{2}} \operatorname{etr}\left[-\frac{1}{2} \Sigma^{-1}\left\{(Z-M)^{\prime}(Z-M)+\delta M^{\prime} D_{r} M\right\}\right]
$$

with $\delta=1 / \tau^{2}$, where etr $(A)$ denotes exponential trace of matrix $A$. The trade-off parameter $\delta$ balances smoothness and goodness of fit.

The unknown parameters are estimated through the marginal likelihood, see, e.g., Yanagimoto and Yanagimoto (1987). First, define the $n \times n$ orthonormal matrix $F=$ $\left[\boldsymbol{f}_{1}, \ldots, \boldsymbol{f}_{n}\right]$ with $\boldsymbol{f}_{1}, \ldots, \boldsymbol{f}_{\boldsymbol{r}}$ of $(3)$ and the normalized eigenvectors $\boldsymbol{f}_{\boldsymbol{r}+1}, \ldots, \boldsymbol{f}_{\boldsymbol{n}}$ of $D_{\boldsymbol{r}}$ corresponding to the eigenvalues $\lambda_{r, r+1}, \ldots, \lambda_{r, n}$. Then, transform $Z$ and $M$ as $F^{\prime} Z=\tilde{Z}=$ $\left[\tilde{\boldsymbol{z}}_{1}, \ldots, \tilde{\boldsymbol{z}}_{n}\right]$ and $F^{\prime} M=\tilde{M}=\left[\tilde{\boldsymbol{\mu}}_{1}, \ldots, \tilde{\boldsymbol{\mu}}_{n}\right]$. Next, factor the entire likelihood $L_{r}(\Gamma, \delta, \Sigma)$ into the product of the residual and the partial likelihoods as

$$
\begin{aligned}
R L_{r}(\Gamma, \Sigma)= & |2 \pi \Sigma|^{-\frac{r}{2}} \exp \left[-\frac{1}{2} \sum_{i=1}^{r}\left(\tilde{\boldsymbol{z}}_{i}-\tilde{\boldsymbol{\mu}}_{i}\right)^{\prime} \Sigma^{-1}\left(\tilde{\boldsymbol{z}}_{i}-\tilde{\boldsymbol{\mu}}_{i}\right)\right], \\
P L_{r}(\delta, \Sigma)= & \delta^{-\frac{(n-r) p}{2}}|2 \pi \Sigma|^{-(n-r)} \prod_{i=r+1}^{n} \lambda_{r, i}^{\frac{p}{2}} \\
& \times \exp \left[-\frac{1}{2} \sum_{i=r+1}^{n}\left\{\left(\tilde{\boldsymbol{z}}_{i}-\tilde{\boldsymbol{\mu}}_{i}\right)^{\prime} \Sigma^{-1}\left(\tilde{\boldsymbol{z}}_{i}-\tilde{\boldsymbol{\mu}}_{i}\right)+\delta \lambda_{r, i} \tilde{\boldsymbol{\mu}}_{i}^{\prime} \Sigma^{-1} \tilde{\boldsymbol{\mu}}_{i}\right\}\right] .
\end{aligned}
$$

The regression coefficient matrix $\Gamma$ is estimated as $\widehat{\Gamma}=F_{r}^{\prime} Y$ by maximizing $R L_{r}(\Gamma, \Sigma)$ where $F_{r}$ is defined in (3). The partial marginal likelihood $P M L_{r}(\delta, \Sigma)$ obtained by integrating out $\boldsymbol{\mu}_{r+1}, \ldots, \boldsymbol{\mu}_{n}$ from $P L_{r}(\delta, \Sigma)$ is written by

$$
P M L_{r}(\delta, \Sigma)=|2 \pi \Sigma|^{-\frac{(n-r)}{2}} \prod_{i=r+1}^{n}\left(\frac{\delta \lambda_{r, i}}{1+\delta \lambda_{r, i}}\right)^{\frac{p}{2}} \operatorname{etr}\left[-\frac{1}{2} \Sigma^{-1} Z^{\prime}\left\{I-\left(I+\delta D_{r}\right)^{-1}\right\} Z\right] .
$$

The estimator of $\Sigma$ is obtained by maximizing $P M L_{r}(\delta, \Sigma)$ for given $\delta \geq 0$ :

$$
\widehat{\Sigma}(\delta) \equiv Z^{\prime}\left\{I-\left(I+\delta D_{r}\right)^{-1}\right\} Z /(n-r) .
$$

Then, the smoothing parameter $\delta$ itself is estimated by maximizing

$$
P M L_{r}(\delta, \widehat{\Sigma}(\delta))=(2 \pi e)^{-\frac{(n-r) p}{2}}|\widehat{\Sigma}(\delta)|^{-\frac{(n-r)}{2}} \prod_{i=r+1}^{n}\left(\frac{\delta \lambda_{r, i}}{1+\delta \lambda_{r, i}}\right)^{\frac{p}{2}} .
$$

The estimator of $\delta$ is written as $\widehat{\delta}$. Finally, we arrive at the predictor of $Z$ as

$$
\widehat{M}=\left(I+\widehat{\delta} D_{r}\right)^{-1} Z
$$

\section{Application to the color images}

The multivariate model for setting $p=3$ in Section 2 is applied to smoothing of noisy color images. The elements of an observed vector $z_{s}^{\prime}=\left(z_{s 1}, z_{s 2}, z_{s 3}\right)$ denote the intensity of 


\section{KUSANOBU}

red, green and blue lights. The spatial smoothing of the image is carried out by the following method. In the color image of size $n \times m$, pick up the $t$-th column data $z_{1}, \ldots z_{n}$, see Figure 1. We suppose that these data follow the Bayes model (2) and get the estimators $\widehat{z}_{1}, \ldots, \widehat{z}_{n}$ by (5). Repeat this smoothing procedure along the vertical direction for $t=1, \ldots, m$. Then we obtain the smoothed image. Next, in the obtained image, pick up the $s$-th column data $\widehat{z}_{1}, \ldots \widehat{z}_{m}$, see Figure 2. We suppose that these data follow the Bayes model, and get the estimators $\widehat{\widehat{z}}_{1}, \ldots, \widehat{\widehat{z}}_{m}$. Repeat this smoothing procedure along the horizontal direction for $s=1, \ldots, n$. Then we obtain the re-smoothed image along the vertical direction after the smoothing by the horizontal direction. This procedure is defined as $\mathrm{Y}-\mathrm{X}$ smoothing. The procedure such as the horizontal smoothing after the vertical smoothing is defined as $\mathrm{X}-\mathrm{Y}$ smoothing.

The proposed methods for the first to third order difference matrix are applied to restoring the noisy test image Lenna and removing stripe noise in water area of a TM true color image.

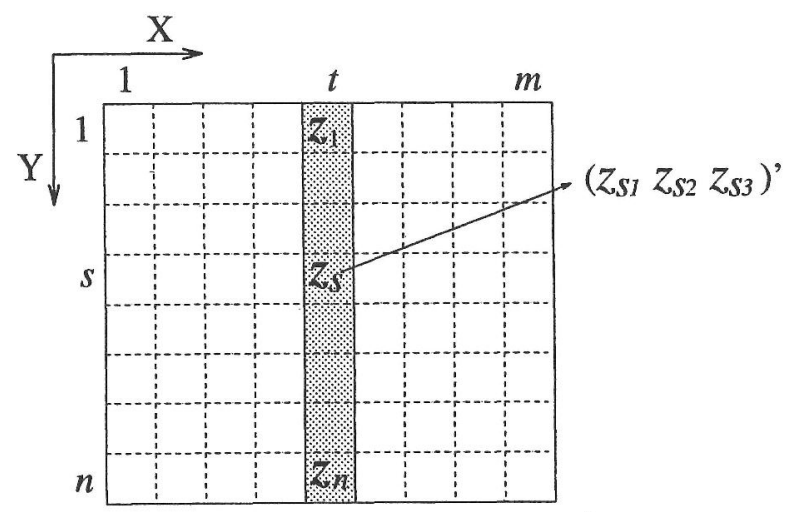

Figure 1. The $t$-th column data in the color image of size $n \times m$

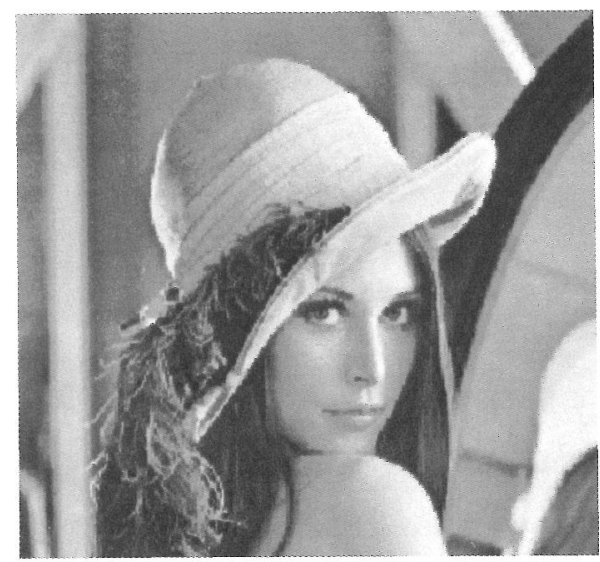

Figure 3. Original Lenna

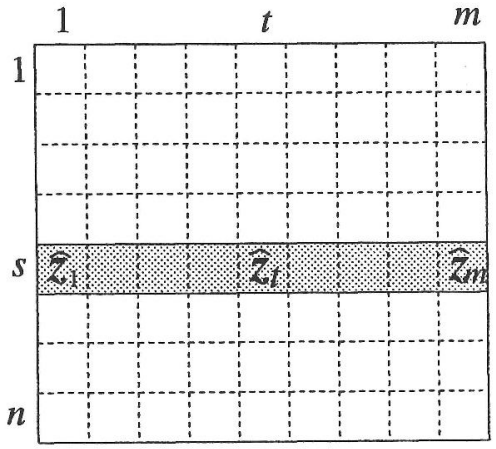

Figure 2. The $s$-th row data in the smoothed image along the vertical direction

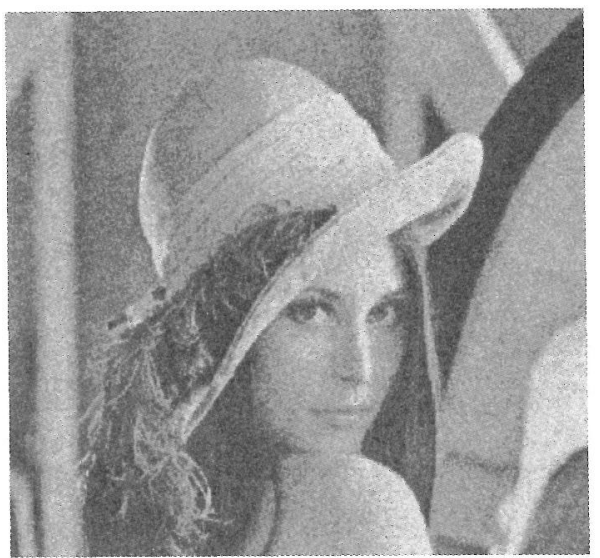

Figure 4. Noisy version with $\frac{1}{4} \widehat{\Xi}$ 
Table 1. RSSE for normal errors with variance $\frac{1}{4} \widehat{\Xi}$

\begin{tabular}{|c|c|c|c|c|c|c|c|c|}
\hline & \multicolumn{3}{|c|}{ Multivariate Bayes Models } & \multicolumn{3}{|c|}{ Univariate Bayes Models } & \multirow{2}{*}{$\begin{array}{l}\text { Lowpass } \\
\text { Filtering }\end{array}$} & \multirow{2}{*}{$\begin{array}{l}\text { Median } \\
\text { Filtering }\end{array}$} \\
\hline & 1st order & 2nd order & 3rd order & 1st order & 2nd order & 3rd order & & \\
\hline $\mathrm{X}-\mathrm{Y}$ & .8975 & .6960 & .7666 & .5689 & .6682 & .7396 & .6603 & .7352 \\
\hline $\mathrm{Y}-\mathrm{X}$ & .8861 & .6649 & .7134 & .5709 & .6464 & .7008 & & \\
\hline
\end{tabular}

Table 2. RSSE for normal errors with variance $\widehat{\Xi}$

\begin{tabular}{|c|c|c|c|c|c|c|c|c|}
\hline & \multicolumn{3}{|c|}{ Multivariate Bayes Models } & \multicolumn{3}{|c|}{ Univariate Bayes Models } & \multirow{2}{*}{$\begin{array}{l}\text { Lowpass } \\
\text { Filtering }\end{array}$} & \multirow{2}{*}{$\begin{array}{c}\text { Median } \\
\text { Filtering }\end{array}$} \\
\hline & 1st order & 2 nd order & 3rd order & 1st order & 2nd order & 3rd order & & \\
\hline $\mathrm{X}-\mathrm{Y}$ & .5019 & .4270 & .4721 & .3535 & .4085 & .4541 & .3830 & .5369 \\
\hline $\mathrm{Y}-\mathrm{X}$ & .4876 & .3847 & .4101 & .3431 & .3778 & .4057 & & \\
\hline
\end{tabular}

\subsection{Example: Lenna}

The test image Lenna is the standard image for image processing. Figure 3 shows the original Lenna of size $520 \times 412$. Its sample variance-covariance matrix is

$$
\widehat{\Xi}=\left(\begin{array}{lll}
2374.0 & 2270.6 & 1130.2 \\
2270.6 & 2810.1 & 1649.4 \\
1130.2 & 1649.4 & 1165.8
\end{array}\right)
$$

Two simulated images are generated by adding two types of normal error, one with variance $\frac{1}{4} \widehat{\Xi}$ and the other with variance $\widehat{\Xi}$.

The simulated image for normal errors with variance $\frac{1}{4} \widehat{\Xi}$ (Figure 4) is smoothed by the first to third order multivariate smoothing (Figures 5-10). We compare the multivariate smoothing with some other method for a monochrome image. The compared methods are a univariate Bayes model (Nishii, Yanagimoto and Kusanobu (1997)), lowpass filtering which cuts off high frequency at 20 percent, and median filtering which uses 5-by-5 neighborhood. The quantitative comparisons of their performances are assessed in terms of the sum of squared errors (SSE) through Mahalanobis distance. Define the original, simulated and smoothed image as $\mu_{i}, z_{i}$ and $\widehat{z}_{i}(i=1, \ldots, 520 \times 412)$, respectively, then the ratio of SSE (RSSE) is calculated by

$$
\sum_{i=1}^{520 \times 412}\left(\widehat{\boldsymbol{z}}_{i}-\boldsymbol{\mu}_{i}\right)^{\prime} \widehat{\Xi}^{-1}\left(\widehat{\boldsymbol{z}}_{i}-\boldsymbol{\mu}_{i}\right) /\left(z_{i}-\boldsymbol{\mu}_{i}\right)^{\prime} \widehat{\Xi}^{-1}\left(z_{i}-\boldsymbol{\mu}_{i}\right) .
$$

The values of RSSE for the noisy images with variance $\frac{1}{4} \widehat{\Xi}$ are listed at Table 1 . The values of RSSE for Y-X smoothing is smaller than for X-Y smoothing in the multivariate and univariate cases. The second order smoothing takes the smallest value in the multivariate models. On the other hand, the first order smoothing (Figure 11) takes the smallest value in the univariate models.

For visual quality, the procedures of Bayes models perform better in a homogeneous area. However, the edges in the image of the third order multivariate smoothing (Figures $9,10)$ are blurred. The first direction of spatial smoothing is more effective than the second direction because the multivariate Y-X smoothings enhance the vertical lines (Figures 


\section{KUSANOBU}

6,8,10). Figures 11 and 12 are the image by the univariate Bayes model. In Figures 13 and 14 obtained by lowpass filtering and median filtering, the edges are preserved, but noise still remains.

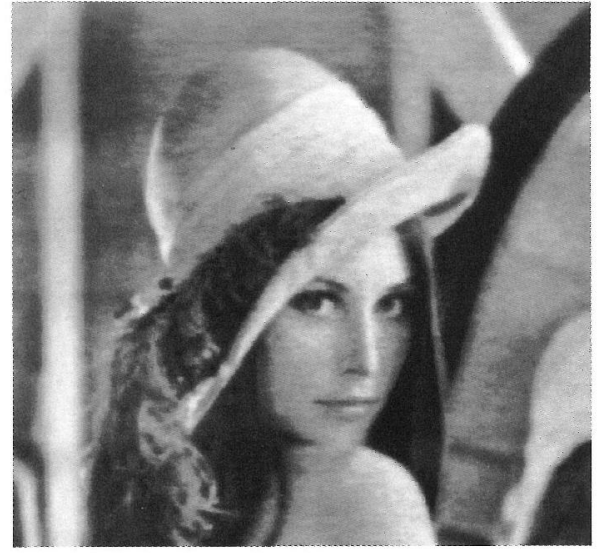

Figure 5. 1st order multivariate $\mathrm{X}-\mathrm{Y}$ smoothing

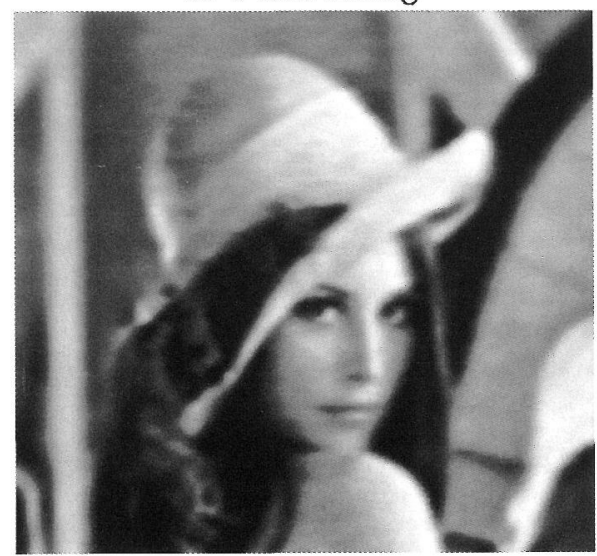

Figure 7. 2nd order multivariate $\mathrm{X}-\mathrm{Y}$ smoothing

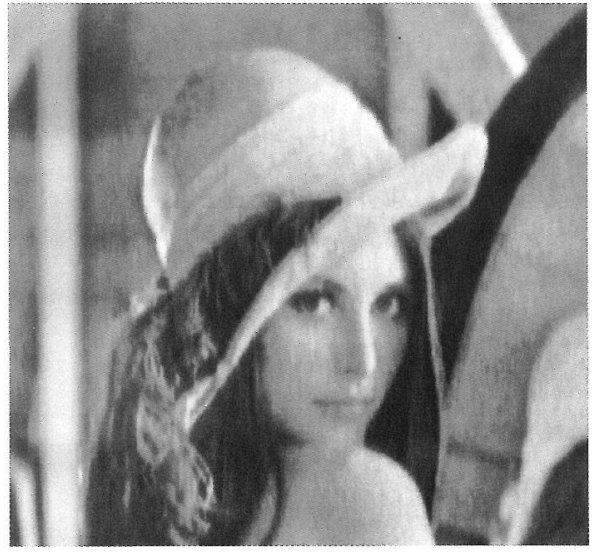

Figure 6. 1st order multivariate Y-X smoothing

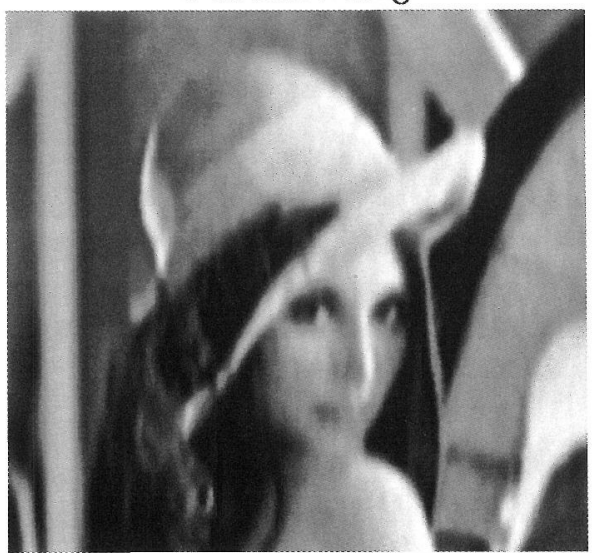

Figure 8. 2nd order multivariate Y-X smoothing

Next, the noisy image to which the normal errors with variance $\widehat{\Xi}$ (Figure 15 ) are added is simulated. The value of RSSE by the second order smoothing (Figure 17) is smaller than the image by the first order smoothing (Figure 16) at Table 2. However, Figure 15 is not better than Figure 16 for visual quality. The new noise line due to the initial smoothing direction appears in the obtained image by the first order $\mathrm{Y}-\mathrm{X}$ univariate smoothing (Figure 18). 
Multivariate Bayes Regression Models for Smoothing of Color Images

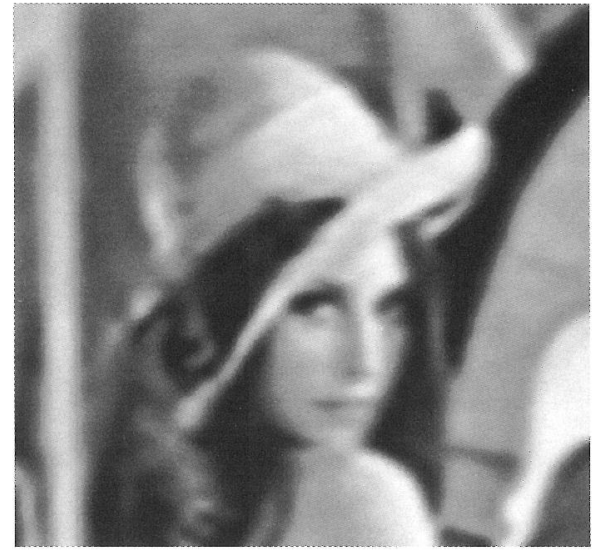

Figure 9. 3rd order multivariate $\mathrm{X}-\mathrm{Y}$ smoothing

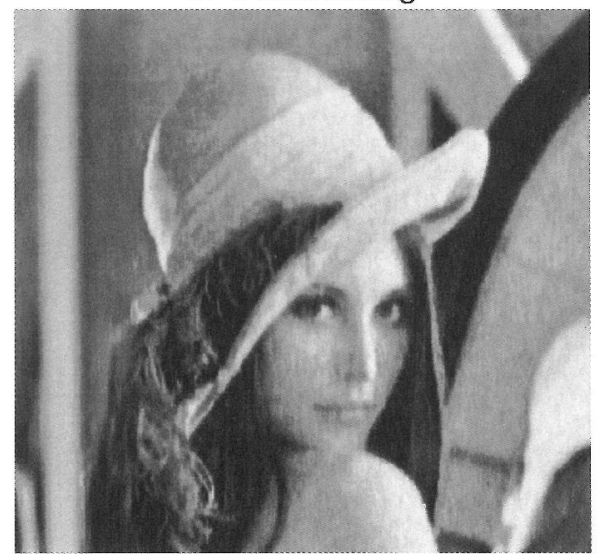

Figure 11. 1st order univariate Y-X smoothing

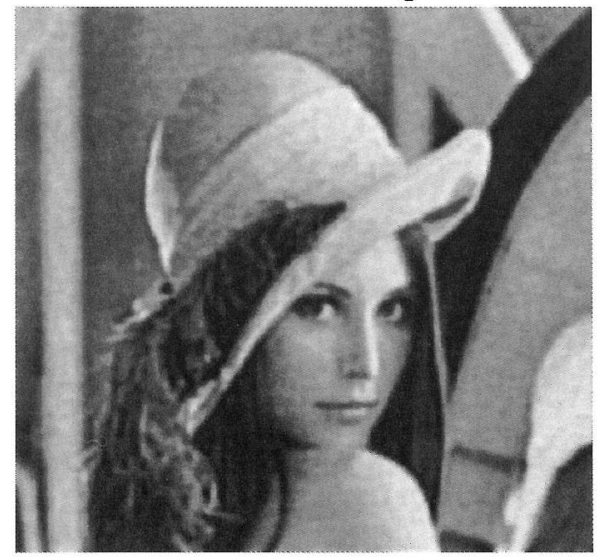

Figure 13. Lowpass filtering

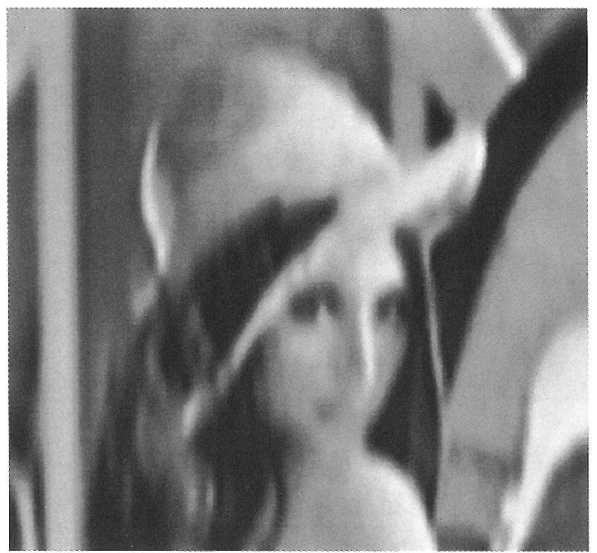

Figure 10. 3rd order multivariate Y-X smoothing

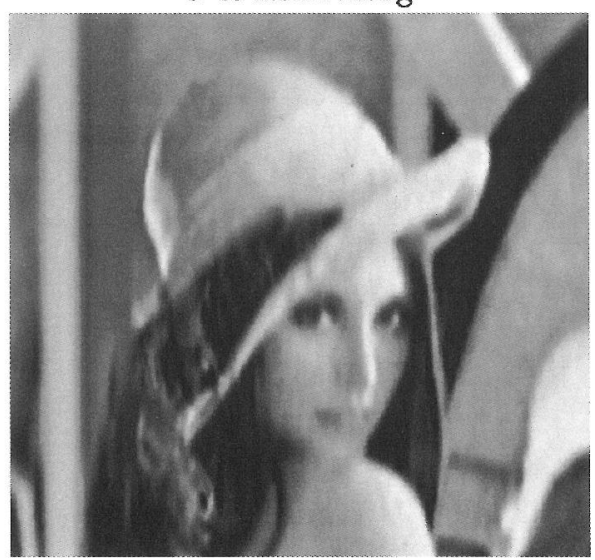

Figure 12. 2nd order univariate Y-X smoothing

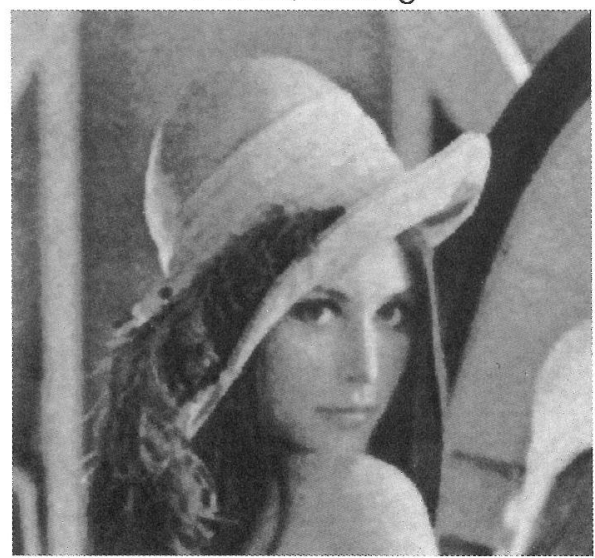

Figure 14. $5 \times 5$ median filtering 


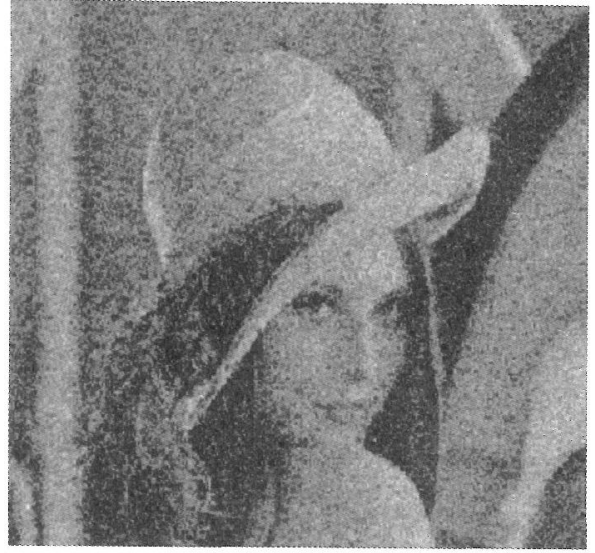

Figure 15 . Noisy version with $\widehat{\Xi}$

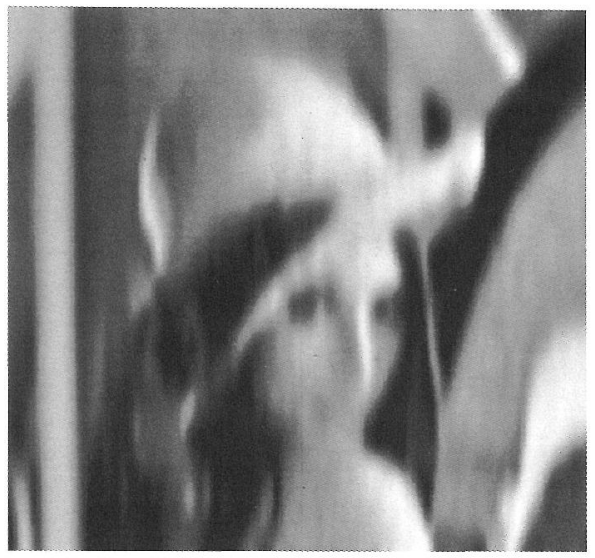

Figure 17. 2nd order multivariate Y-X smoothing

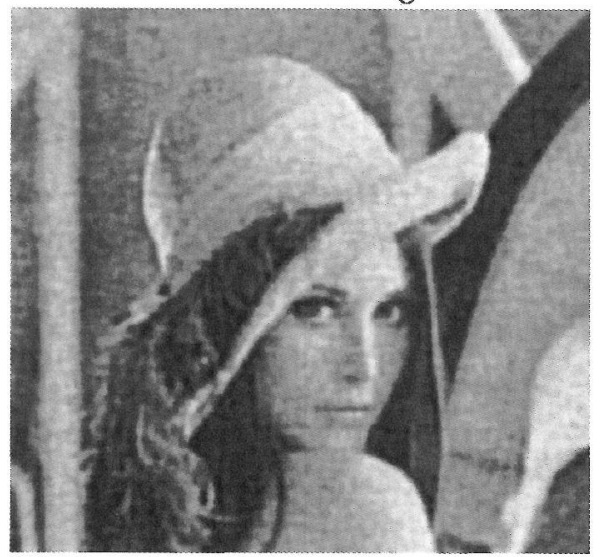

Figure 19. Lowpass filtering

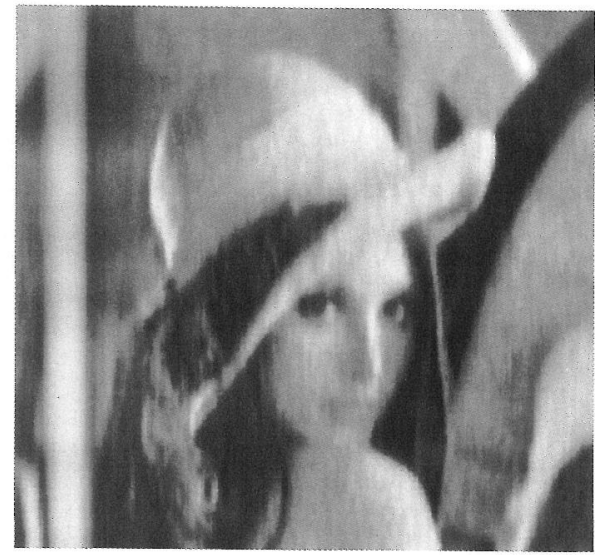

Figure 16. 1st order multivariate Y-X smoothing

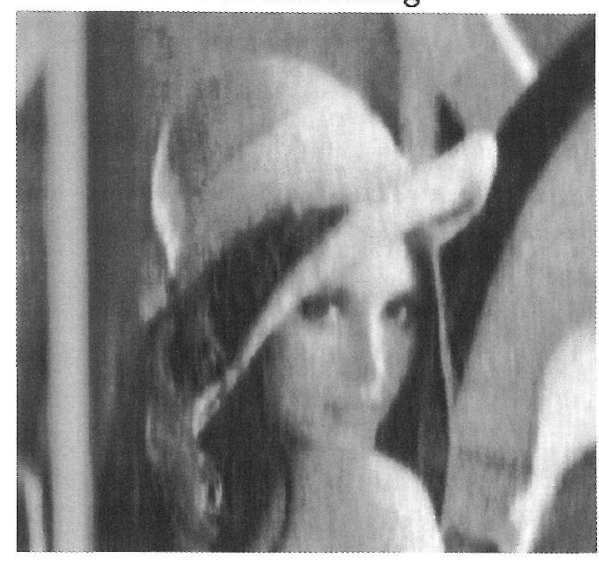

Figure 18. 1st order univariate Y-X smoothing

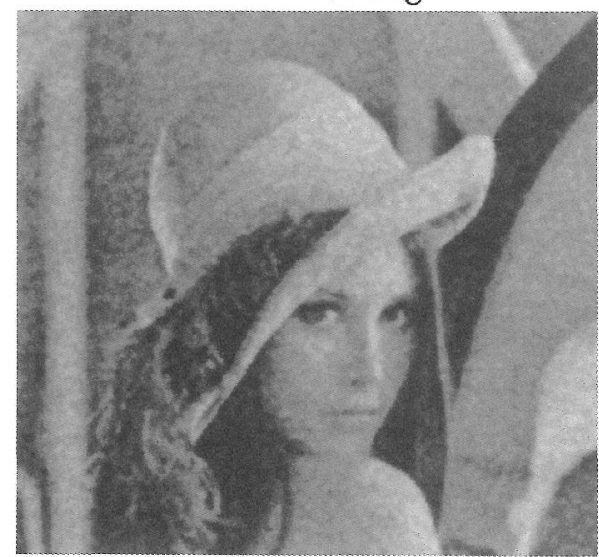

Figure $20.5 \times 5$ median filtering 


\subsection{Example: Landsat TM image of water area}

In this example, we treat the image obtained by the satellite, Landsat 5. Figure 21 is the image composed of the three bands of visible radiation of Landsat TM data of size $355 \times 407$ of Seto Inland Sea, Japan. We apply Y-X smoothing to this image for removing stripe noise.

Figure 22 is the smoothed image of the first order multivariate smoothing. We succeed in reducing the stripe noise at the horizontal direction. However, new stripe noise at the vertical directions due to the initial smoothing direction is observed. Figure 23 results of the second order smoothing. We can see the equal depth in the sea by noise removal (Figure 23). In the third order smoothing (Figure 24), the stripe noise is deleted, but the edge of the island is blurred. In order to remove stripe noise of TM images, the second order smoothing shows the best performance.

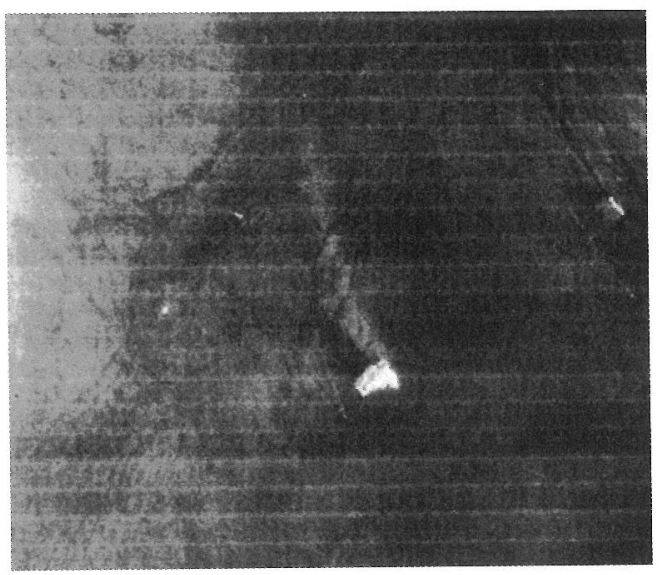

Figure 21. True color TM image of Seto Inland Sea, Japan

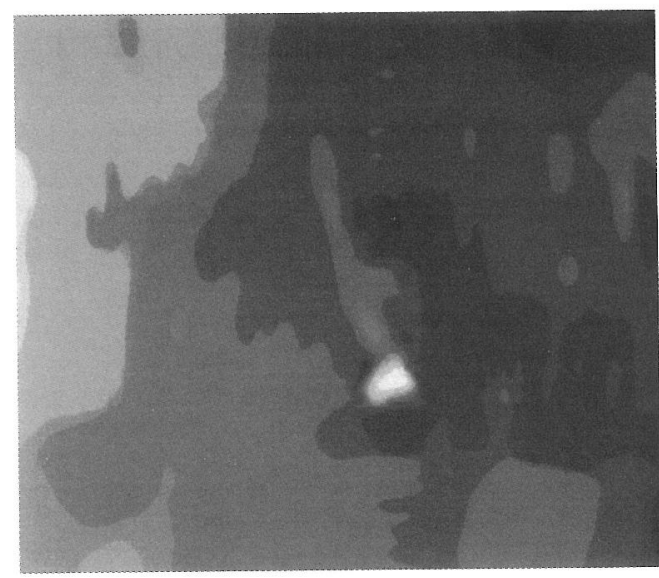

Figure 23. 2nd order multivariate smoothing

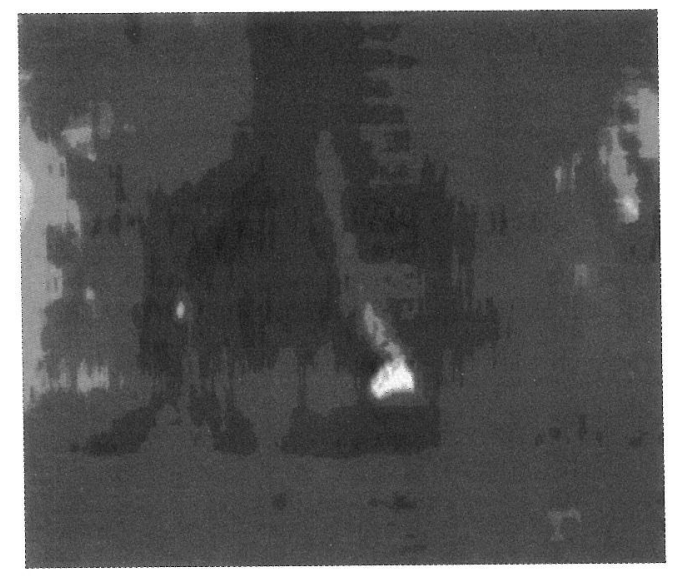

Figure 22. 1st order multivariate smoothing

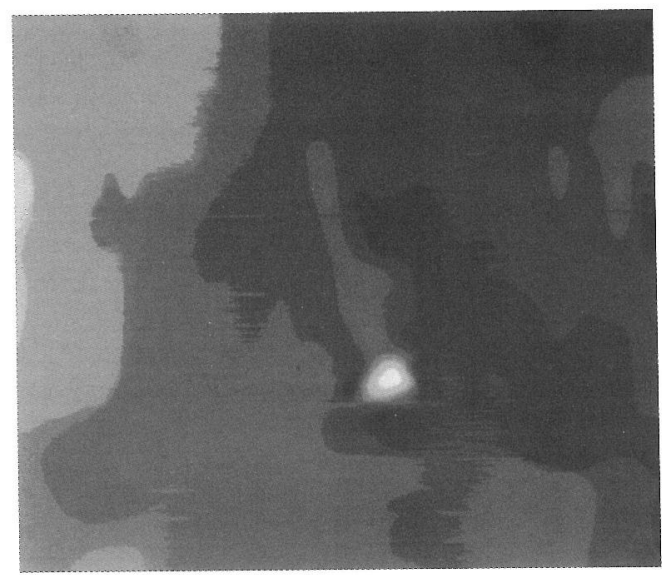

Figure 24. 3rd order multivariate smoothing 


\section{KUSANOBU}

\section{Discussion}

We propose the multivariate Bayes models using the difference matrix $D_{r}, r=1,2,3$, in smoothness prior. If the smoothing parameter $\delta$ is estimated by maximizing the partial marginal likelihood (4), the observations are predicted by simple form (5). These models, which take into account correlations of three monochrome images, can be applied to smoothing of color images.

The features of our methods used for the color smoothing are the following. The values of RSSE by the second and the third order smoothing are small, although the obtained images have a tendency for over-smoothing. RSSE of the univariate Bayes model is smaller than that of the multivariate Bayes model. The spatial smoothing by two directions is applied with the aim of destriping TM image. For spatial smoothing, the final image is affected by the initial smoothing direction. Therefore, in order to remove stripe noise in TM image, horizontal smoothing after vertical smoothing is suitable.

\section{Acknowledgements}

The author would like to thank Professor Ryuei Nishii of Hiroshima University for his helpful comments in this paper. The author also thanks the referees for the careful reading and the useful comments.

\section{REFERENCES}

Karlin, S., Micchelli, C. A. and Rinott, Y. (1986). Multivariate splines: a probabilistic perspective. J. Multi. Anal., 20, 69-90.

Nishii, R., Yanagimoto, T. and Kusanobu, S. (1997). The use of univariate Bayes regression models for spatial smoothing. Comput. Statist. Data Anal., 24, 321-336.

Pan, J.J. and Chang, C.I. (1992). Destriping of Landsat MSS images by filtering techniques. Photogrammetric Engineering \& Remote Sensing, 58, 1417-1423.

Stone, C. J. (1994). The use of polynomial splines and their tensor products in multivariate function estimation. Ann. Statist., 22(1), 118-184.

Yanagimoto, T. and Yanagimoto, M. (1987). The use of marginal likelihood for a diagnostic test for the goodness of fit of the simple linear regression model. Technometrics, 29, 95-101.

(Received May 1998; Revised December 1998) 